\title{
Ability to perform Activities of Daily Living among patients with bipolar disorder in remission
}

\author{
Lone Decker, Conny Träger, Kamilla Miskowiak, \\ Eva Ejlersen Wæhrens, Maj Vinberg
}

\section{ABSTRACT}

Aims: Patients with bipolar disorder often experience disability in terms of cognitive impairments and activity limitations even in remission. However, knowledge is sparse concerning the ability to perform Activities of Daily Living (ADL) during remission. The aim of this study was to (1) investigate the observed and self-reported ability to perform ADL tasks and (2) examine the association between observed and self-reported ability to perform ADL in patients with bipolar disorder in remission. Methods: The observed ADL ability was assessed with the Assessment of Motor and Process Skills, a standardized assessment providing interpretation of ADL ability in

Lone Decker ${ }^{1}$, Conny Träger $^{2}$, Kamilla Miskowiak ${ }^{3}$, Eva Ejlersen Wæhrens ${ }^{4}$, Maj Vinberg ${ }^{5}$

Affiliations: ${ }^{1} \mathrm{MEd}$. Associate Professor, Department of Physiotherapy and Occupational Therapy, Metropolitan University College, Copenhagen, Denmark; ${ }^{2} \mathrm{MD}$, Registrar, Psychiatric Centre Copenhagen, Copenhagen University Hospital, Copenhagen, Denmark; ${ }^{3} \mathrm{PhD}$, Senior Research Psychologist, Psychiatric Centre Copenhagen, Copenhagen University Hospital, Copenhagen, Denmark; ${ }^{4} \mathrm{PhD}$, Senior researcher, The Parker Institute, Copenhagen University Hospital, Bispebjerg and Frederiksberg, Frederiksberg, Denmark, Associate Professor, The Research Initiative for Activity Studies and Occupational Therapy, Institute of Public Health, University of Southern Denmark, Odense, Denmark; ${ }^{5} \mathrm{DMSc}$, Consultant, Associate Professor, Psychiatric Centre Copenhagen, Copenhagen University Hospital, Copenhagen, Denmark.

Corresponding Author: Lone Decker, Sigurdsgade 26, 2200 Copenhagen N, Denmark; Email: lode@phmetropol.dk

Received: 03 July 2017

Accepted: 24 August 2017

Published: 19 September 2017 relation to competence, independence, and normative age expectations. Self-reported ADL ability was assessed with the standardized ADLQuestionnaire. Results: Forty-three patients with bipolar disorder in remission (median age 35 years, range $19-58$ years) were assessed and overall, they displayed decreased observed ADL motor and ADL process ability relative to normative age. They exhibited increased physical effort, clumsiness or fatigue and/or inefficiency, there was concern for safe task performance and one-third may need assistance to live in the community. While participants reported decreased ADL ability, especially within instrumental ADL, they had a tendency towards evaluating themselves as more competent than what was observed. No relationships between measures of observed and self-reported ADL ability were found. Conclusion: Overall, patients with bipolar disorder in remission showed decreased ability to perform ADL. Clinical practice and future studies are recommended to use both observation-based and self-reported assessments of the ability to perform ADL tasks to fully capture disability in bipolar disorder.

Keywords: Activities of Daily Living, ADL-Questionnaire, Assessment of Motor and Process Skills, Functioning, Performance-based assessment, Self-reporting assessment

\section{How to cite this article}

Decker L, Träger C, Miskowiak K, Wæhrens EE, Vinberg M. Ability to perform Activities of Daily Living among patients with bipolar disorder in remission. Edorium J Disabil Rehabil 2017;3:69-79. 


\section{EDORiUM Journals}

Edorium J Disabil Rehabil 2017;3:69-79.

Decker et al.

$* * * * * * * * *$

doi:10.5348/Do5-2017-33-OA-9

\section{INTRODUCTION}

Bipolar disorder is a main cause of decreased functioning worldwide with a prevalence $>2 \%$ [1-4]. However, descriptions of the impact of bipolar disorder on patients' functioning are often hampered by unclear definitions of the concept of functioning [5]. The International Classification of Functioning, Disability and Health (ICF) [6] is recommended as a conceptual model to describe functioning and disability within mental health in general [7] and within bipolar disorder $[5,8,9]$. The majority of research on functioning and disability within bipolar disorder has focused on the component body functions and body structures typically in terms of cognition [5]. Cognitive impairments are well documented within bipolar disorder even in remission $[10,11]$. Fewer studies have addressed the component activities and participation [5] including self-care and domestic life, also termed Activities of Daily Living (ADL) $[6,12]$. The ability to perform ADL tasks is required for independent living and represents the basis for other activities including work and leisure [12]. Personal ADL (PADL) involves tasks such as personal hygiene, eating and reading, whereas Instrumental ADL (IADL) is related to more complex home maintenance tasks e.g., cleaning, cooking, and shopping $[6,12]$. The ability to carry out these daily routines is included in both the Comprehensive ICF Core Set for bipolar disorder and in the Brief ICF Core Set for bipolar disorder [8].

Studies assessing ADL ability indicate that patients with bipolar disorder have limited ability to perform ADL tasks, especially IADL tasks [13-16]. Different types of assessment have been used to evaluate the ADL ability within bipolar disorder but dissimilarities between the results of the assessments have been found. Most studies on ADL have used clinician-rated assessments [17]. However, as it is necessary to also consider the patient's perspective [18] when providing needed interventions to improve functioning, [19, 20] self-reported assessments of ADL ability have also been used [21-23]. However, previous studies suggest that symptom severity and cognitive impairments within bipolar disorder may impact the validity of self-reported data $[14,17,21,22]$ and direct observation is, therefore, considered the most reliable source of information [24]. Observation-based assessments have been used among patients with bipolar disorder $[14,15,25,26]$ however, concerns related to the validity of conducting such assessments in a clinical setting $[27,28]$ and the need for assessment instruments sensitive to change over time have been raised [24,
27]. Further, observation-based instruments based on sufficiently large normative standardization samples have been lacking [27].

Until now, the focus of studies using observationbased assessments of ADL ability within bipolar disorder has been on the relationship between cognitive impairments and ADL task performance rather than on the competence of performing ADL on itself. Previous studies have found an association between cognitive impairments and decreased ADL task performance $[14,15,26]$. However, a recent meta-analysis of the association between cognition and the ability to perform activities recommend assessments of both areas and better understanding of the validity and usability of performance-based measures [17]. Thus, studies regarding the use of observation-based assessments among patients with bipolar disorder in remission are lacking and to the best of our knowledge the association between self-reported and observed ADL ability within this group has not previously been studied.

Therefore, the aim of this study was to

- investigate the observed and self-reported ability to perform ADL tasks, and

- examine the association between observed and self-reported ability to perform ADL in remitted patients with bipolar disorder reporting subjective cognitive complaints.

\section{MATERIALS AND METHODS}

\section{Participants}

Participants with bipolar disorder (as defined by ICD10 diagnostic criteria) [29] were recruited from the Clinic for Affective Disorders, Psychiatric Centre Copenhagen, between February and October 2015. The clinicians were encouraged to ask all patients between 18 and 60 years with bipolar disorder in remission to participate. Mood symptoms were rated with the Hamilton Depression Rating Scale (HDRS-17) [30] and the Young Mania Rating Scale (YMRS) [31]. Full remission was defined by HDRS17 and YMRS scores $\leq 7$ for at least a week. Afterwards, the patients were asked to complete the Cognitive Complaints in Bipolar Disorder Rating Assessment (COBRA) questionnaire [32]. Patients having subjective complaints of cognitive difficulties of moderate to severe degree as reflected by COBRA scores $\geq 13$ were included [33].

Exclusion criteria were current substance or alcohol abuse, schizophrenia, schizoaffective disorder or major psychical disease. This was initially determined by reading patients' case files. Further, the occupational therapist conducting the Assessment of Motor and Process Skills (AMPS) evaluations assed the impact of any physical disease or physical complaints on ADL task performance. If it was determined that the physical disease was the 


\section{EDORIUM Journals}

Edorium J Disabil Rehabil 2017;3:69-79.

Decker et al.

dominating reason for any ADL problems, patient was excluded from the study.

Participants continued their usual medication and were permitted to take benzodiazepines corresponding to $\leq 22.5 \mathrm{mg}$ oxazepam.

Results on cognition derived from the same study population have been reported in another paper. In summary, we showed that patients' subjective cognitive complaints did not correlate with ADL ability. However, we observed a correlation between slower processing speed (assessed with objective tests) and poorer ADL process ability and a trend towards a correlation between slower processing speed and poorer ADL motor ability. Further, this processing speed deficit was also associated with lower level of ADL ability, as reflected by greater need of assistance to live in the community. Slowed processing speed in bipolar disorder may therefore be a key indicator for patients' general ability to live independently in the community [34].

\section{Assessments of ADL ability}

Observation-based ADL ability was assessed using the Assessment of Motor and Process Skills (AMPS) which is a standardized observation-based assessment providing measures of the quality of ADL task performance. During an interview, the participant selects two ADL tasks to perform. The tasks have to be well known, meaningful and challenging for the person to perform [12]. In the manual, there are currently 125 standardized tasks calibrated in terms of severity of the task. These tasks can to some extent be varied to allow for individual and cultural differences, while still respecting the standardization [35]. Following the observation, two domains of performance are evaluated for each task: ADL motor skills (16 items) and ADL process skills (20 items). The quality of each ADL skill is scored on a four-point ordinal scale [12]. The scores for both tasks are entered into a many-faceted Raschbased computer software program [36] that converts the ordinal data into two overall linear measures of ADL motor and ADL process ability adjusted for task difficulty and rater severity. The ADL motor ability measure is an indication of how much effort or clumsiness the person demonstrates when performing ADL tasks. The ADL process ability measure indicates how timely and well organized the person was during the observation. Both scales also reflect safety (no risk of personal injury or environmental damage) and independence (no need for physical or verbal assistance) in ADL task performance. The current software is based on the international AMPS database that includes data from $>13,000$ calibrated testers and >150,000 individuals worldwide [12]. The AMPS ADL ability measures are found valid and reliable across diagnostic groups, including mental disorders containing bipolar disorder [37-41], culture, age and gender [12]. The AMPS has a high inter-rater reliability and is found to be sensitive over time to changes in ADL ability [12].

Self-reported ADL ability was assessed using the standardized ADL-Questionnaire (ADL-Q). The participant is requested to evaluate their perceived ADL ability within the past 24 hours in 31 PADL tasks and within the last seven days in 16 IADL tasks. There are seven response categories reflecting efficiency, effort/fatigue, safety, and independence, including the possibility of marking IADL tasks 'not relevant in my daily life' [42]. Studies support that ADL-Q can be used to generate valid and reliable measures of self-reported quality of ADL task performance among patients with long-term or chronic diseases such as rheumatism [43] and chronic obstructive pulmonary disease [44]. No studies have previously used ADL-Q within mental disorders, but the interviewbased equivalent, the ADL-I, has been used in persons with depression and schizophrenia [45] confirming the relevance of the $47 \mathrm{ADL}$ tasks in this population.

\section{Design and procedures}

This study applied a cross-sectional design to investigate ADL ability among patients with bipolar disorder in remission reporting cognitive impairments. The participants were screened with HDRS-17, YMRS and COBRA by one of the specialists in psychiatry at the Copenhagen Clinic for Affective Disorders, The Capital Region of Copenhagen, at the Psychiatric Centre Copenhagen. Demographic data collection and introductions to the ADL-Q were conducted within a week after mood screening by a medical student at the Clinic for Affective Disorders. The AMPS evaluations were planned to be conducted within 14 days after the initial screening with HDRS-17 and YMRS, to ensure that the participants were still in remission. The AMPS was conducted by an AMPS-calibrated occupational therapist and administered according to standardized procedures $[12,35]$ in patients' homes. The interview and observation took approximately one hour. The AMPS rater was blinded for the result of the ADL-Q. Before the AMPS observation, the AMPS rater phoned the participant in order to clarify which tasks he or she normally performs and would be willing to perform while observed. Since the participants were in remission, lived in their own home and were thus expected to be relatively well functioning, they were offered IADL tasks (e.g. home maintenance, cooking meals) calibrated as average, harder or much harder on the AMPS process task challenge hierarchy [35]. This was done to avoid ceiling effects.

\section{Ethical considerations}

Approval from the Danish Data Protection Agency was obtained (journal no. 03238 (I-Suite), ID: RHP-2014039). Procedures were in accordance with the Helsinki Declaration of 1975 . 


\section{EDORIUM Journals}

Edorium J Disabil Rehabil 2017;3:69-79.

Decker et al.

www.edoriumjournals.com/ej/dr

\section{Statistical analysis}

Statistical Package for the Social Sciences version 22 [46] was used for descriptive analyses and nonparametric statistics. Normality of the distribution was tested using Kolmogorov-Smirnoff test. Normally distributed data are presented as mean \pm standard deviation, and nonnormally distributed data are presented as median/range or percentage. The raw ordinal AMPS data was converted into overall linear ADL measures using a Rasch-based computer software program [36]. The two ADL ability measures, ADL motor ability and ADL process ability, are presented in logits (log-odds probability units). The ADL motor and ADL process ability measures were interpreted from a criterion-based perspective related to competence. Higher ADL motor and ADL process ability measures indicate higher ADL ability. The competence cut-off is 2.0 logits for ADL motor ability and 1.0 logits for ADL process ability [12]. Furthermore, an ADL motor ability measure below 1.5 logits and an ADL process ability measure below 1.0 logits combined indicate that the person is more likely to need assistance to live in the community. If the two measures fall within different decision zones, the ADL process ability measure is the strongest predictor of the need for assistance [35]. The percentages of participants within and below competence and independence cut-offs are presented.

In addition, a person's ADL ability can be interpreted from a norm-based perspective by comparing the person's ADL ability measures to the expected range of ADL ability measures for well, typically-developing persons of the same age [35]. These results are presented as number of participants above/below normative mean and within/ outside the $95 \%$ confidence interval (CI) in a stacked bar chart.

The raw ordinal ADL-Q data was assigned to one of four categories: $3=$ competent, $2=$ using extra time/effort, $1=$ need for help/safety risk or $0=$ unable to perform. If $\mathrm{a}$ task was marked 'Not relevant' a fifth category was formed. This was done to prepare conversion of ordinal scores into linear measures of self-reported ability to perform ADL using the Rasch computer software program Winsteps ${ }^{\circledR}$ version 3.68.2 [47]. These self-reported ADL ability measures are, similar to the AMPS ADL ability measures, expressed in logits. [42]. The frequencies of each of the five categories are presented as percentages in a stacked bar chart. As the ADL-Q measures were not normally distributed, the Spearman rank order test was used to investigate the correlations between the overall measures of observed ADL ability (AMPS) and self-reported ADL ability (ADL-Q). In this study, a correlation coefficient $\geq 0.50$ was considered the minimum as evidence of acceptable correlation between measures. Calculations of the coefficients of determination $\left(\mathrm{r}_{\mathrm{s}}{ }^{2}\right)$ were done to determine the extent of shared variance between the measures of self-reported and observed ADL ability. The correlations between overall measures of self-reported and observed ADL ability are presented in scatterplots.

\section{RESULTS}

Overall, 70 patients fulfilled COBRA questionnaire and 47 patients (67\%) had a score above 13 . Two were excluded; one due to current substance abuse and one due to a comprehensive surgery. Two patients dropped out after cognitive testing and fulfilling questionnaires due to unwillingness to participate in the AMPS evaluation in their own home. Thus, a total of 43 patients participated. However, one participant was excluded from the results involving $\mathrm{ADL}-\mathrm{Q}$ due to not being in remission at the time of the ADL-Q.

Participants' demographic, clinical characteristics, and self-reported (ADL-Q) and observed (AMPS) ADL ability measures are presented in Table 1 . The sample had a median age of 35 years and $30(70 \%)$ were women. Nineteen (44\%) of the participants were either working or in education. They were all living in their own home, 22 (51\%) with a partner. In total, 15 of the participants had physical disease or complaints that potentially could affect their ADL ability. These were mainly musculoskeletal complaints and migraine/headache. Only three patients had physical complaints at the time of the AMPS observation that the rater judged affected their ADL ability. However, physical complaints were not the dominant reason for ADL problems in any of these cases.

\section{Observed ability to perform ADL tasks}

The participants' AMPS ADL motor and ADL process ability measures in relation to competence cut-off and independence cut-off are presented in Table 2. Although the participants' mean ADL motor and ADL process ability measures were at the 2.o logit competence cut-off on the ADL motor scale and just above the 1.o logit cut-off on the ADL process scale, respectively, the results showed that almost one-third had an ADL motor ability measure below the ADL motor competence cut-off indicating increased physical effort, clumsiness or fatigue and potential risk of unsafe task performance. Likewise, almost one-third had an ADL process ability measure below the ADL process competence cut-off, indicating decreased disorganization or undesirable use of time, space or objects and potential risk of unsafe task performance. Five patients (12\%) had both ADL motor and ADL process ability measures below the competence cut-offs. As for independence, two of the participants had an ADL motor ability measure below the motor independence cut-off at 1.5 logits, whereas almost one-third had an ADL process ability measure below the process independence cut-off at 1.0 logit, indicating that almost one third of the included participants may need assistance to live in the community. However, only one participant had ADL ability measures below both independence cut-offs. 


\section{EDORIUM Journals}

Edorium J Disabil Rehabil 2017;3:69-79.

Decker et al.

www.edoriumjournals.com/ej/dr

Table 1: Participant characteristics, obtained observed Activities of Daily Living (ADL) motor and ADL process ability measures and self-reported ADL ability measure

\begin{tabular}{|c|c|}
\hline \multicolumn{2}{|l|}{$n=43$} \\
\hline Age, in years (median/range) & $35(19-58)$ \\
\hline Gender (female) & $69.8(\%)$ \\
\hline Living alone/cohabiting & $48.8 / 51.2(\%)$ \\
\hline Housing own home & $100(\%)$ \\
\hline Children/no children & $46.5 / 53.5(\%)$ \\
\hline Years of education (median/range) & $15(9-21)$ \\
\hline Employed or independent & $18.6(\%)$ \\
\hline In education & $25.6(\%)$ \\
\hline Unemployment fund benefit recipient & $4.7(\%)$ \\
\hline Social security recipient & $11.6(\%)$ \\
\hline Sick leave owing to illness & $25.6(\%)$ \\
\hline Disability pensioner & $14(\%)$ \\
\hline Age at onset (median/range) & $21(13-48)$ \\
\hline Bipolar disorder type I/II & $48.8 / 51.2(\%)$ \\
\hline Months in remission (median/range) & $4(0.25-43)$ \\
\hline Total number of episodes (median/range) & $13(3-85)$ \\
\hline $\begin{array}{l}\text { Number of hospitalizations (median/ } \\
\text { range) }\end{array}$ & $1(0-10)$ \\
\hline Daily use of medication & $95 \cdot 3(\%)$ \\
\hline Number of medications (median/range) & $2(0-4)$ \\
\hline Lithium & $44.2(\%)$ \\
\hline Anticonvulsants & $62.8(\%)$ \\
\hline Antidepressants & $18.6(\%)$ \\
\hline Antipsychotics & $34.9(\%)$ \\
\hline Benzodiazepines & $7.0(\%)$ \\
\hline HDRS-17 score (median/range) & $3(0-7)$ \\
\hline YMRS score (median/range) & $2(0-7)$ \\
\hline COBRA score (median/range) & $22(13-41)$ \\
\hline $\begin{array}{l}\text { AMPS ADL motor ability measure (mean/ } \\
\text { SD) }\end{array}$ & $2.0 / 0.3$ \\
\hline $\begin{array}{l}\text { AMPS ADL process ability measure } \\
(\text { mean/SD) }\end{array}$ & $1.1 / 0.2$ \\
\hline $\begin{array}{l}\text { ADL-Q ability measure }(n=42) \text { (median/ } \\
\text { range) }\end{array}$ & $4.7(1.7-8.0)$ \\
\hline
\end{tabular}

Abbreviations: HDRS-17: Hamilton Depression Rating Scale 17 items, YMRS: Young Mania Rating Scale, COBRA: Cognitive Complaints in Bipolar Disorder Rating Assessment, AMPS: Assessment of Motor and Process Skills, ADL-Q: ADLQuestionnaire
Table 2: Distribution of Activities of Daily Living (ADL) motor and ADL process ability measures in relation to competence cut-off and independence cut-off according to the Assessment of Motor and Process Skills (AMPS) among patients with bipolar disorder

\begin{tabular}{|c|c|c|}
\hline$n=43$ & $\begin{array}{c}\text { ADL motor ability } \\
\text { n (\%) }\end{array}$ & $\begin{array}{l}\text { ADL process } \\
\text { ability n (\%) }\end{array}$ \\
\hline & $\begin{array}{l}\text { Competence cut- } \\
\text { off: } 2.0 \text { logits } \\
\text { Independence cut- } \\
\text { off: } 1.5 \text { logits }\end{array}$ & $\begin{array}{l}\text { Competence cut- } \\
\text { off: } 1.0 \text { logits } \\
\text { Independence } \\
\text { cut-off: } 1.0 \text { logits }\end{array}$ \\
\hline $\begin{array}{l}\text { Above } \\
\text { competence } \\
\text { cut-off }\end{array}$ & $29(67.4(\%)$ & $30(69.8 \%)$ \\
\hline $\begin{array}{l}\text { Below } \\
\text { competence } \\
\text { cut-off }\end{array}$ & $14(32.6 \%)$ & $13(30.2 \%)$ \\
\hline $\begin{array}{l}\text { Above } \\
\text { independence } \\
\text { cut-off }\end{array}$ & $41(95 \cdot 3 \%)$ & $30(69.8 \%)$ \\
\hline $\begin{array}{l}\text { Below } \\
\text { independence } \\
\text { cut-off }\end{array}$ & $2(4.7 \%)$ & $13(30.2 \%)$ \\
\hline
\end{tabular}

Abbreviations: AMPS: Assessment of Motor and Process Skills, ADL: Activities of Daily Living

\section{ADL motor and ADL process ability in relation to age expectations}

The distributions of ADL motor and ADL process ability measures in relation to normative age-matched expectations are presented in Figure 1. As for ADL motor ability, the majority ( $\mathrm{n}=36,84 \%)$ had an ADL motor ability below the mean of healthy age-matched peers, and three (7\%) of these had an ADL motor ability below the 95\% CI (i.e., < 2 SD below the normative mean). Seven participants (16\%) had an ADL motor ability above the normative age-matched mean. All participants had an ADL process ability measure below the mean of healthy, age-matched peers and two (5\%) of these had an ADL process ability measure below the $95 \%$ CI. One participant had both an ADL motor ability measure and an ADL process ability measure below the $95 \% \mathrm{CI}$. Thus, while the majority of participants had ADL ability measures within the expected range of ADL ability for healthy persons of the same age, they were at the lower end of the spectrum. Furthermore, the mean ADL motor ability measure of the study sample (median: 35 years, range: $19-58$ years) is equivalent to the mean ADL motor ability measure for healthy persons between 60 and 64 years (mean: 2.1, SD: 0.4). Similarly, the mean ADL process ability measure for the study sample is equivalent to that of healthy people older than 85 years (mean: 1.3, SD: 0.4) [35]. 


\section{Self-reported ability to perform ADL tasks}

As seen in Figure 2, most participants reported themselves as being competent within the PADL domain. However, for the task 'Reading' approximately 50\% reported themselves not being competent. One participant was unable to perform the task. Two persons reported the task was not relevant. Regarding the task 'Writing by hand' approximately 30\% reported the use of extra time or increased effort. In relation to the communication tasks 'Calling for attention', 'Taking part in a conversation' and 'Using the phone' $15-20 \%$ of the participants did report the use of extra time or increased effort. Within the IADL domain, 20-50\% reported problems related to domestic tasks such as cooking, shopping, cleaning, washing clothes and driving. In the tasks 'Weekly heavy cleaning', 'Daily light cleaning', 'Weekly/large quantity shopping', 'Making plans for shopping', 'Cooking a hot meal' and 'Driving car' a few participants (2-4\%) reported inability to perform the task or a safety risk.

\section{Correlations between measures of observed and self-reported ability to perform ADL}

As seen from Figure 3, no correlation between observed and self-reported ability to perform ADL tasks was found either for ADL-Q and AMPS ADL motor ( $\mathrm{r}=$ $0.001, \mathrm{p}=0.90)$ or ADL-Q and AMPS ADL process $(\mathrm{r}=$ o.009, $\mathrm{p}=0.55$ ) ability measures.

\section{DISCUSSION}

The overall purpose of this study was to explore the ability to perform ADL among patients with bipolar disorder in remission reporting subjective cognitive complaints. Overall, participants in this study had observed and self-reported decreased ADL ability. In fact, their mean ADL motor ability (median: 35 years, range: 19-58 years) was equivalent to that of healthy persons at 60-64 years and their mean ADL process ability was equivalent to healthy persons $>85$ years old [12]. This study is the first to report on both observation-based and self-reported measures of ADL ability among patients with bipolar disorder in remission and no correlation between the two measures was found.

One-third of the included patients with bipolar disorder in remission had an observed ADL motor ability below the AMPS ADL motor competence cut-off

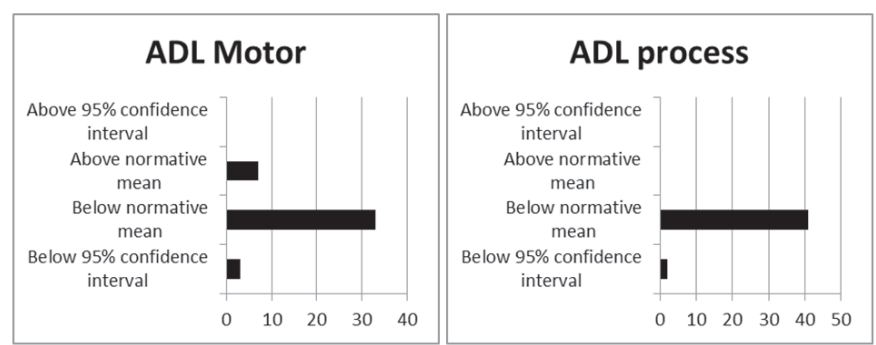

Figure 1: Distribution of Activities of Daily Living (ADL) motor and ADL process ability measures in relation to normative age expectations among patients with bipolar disorder $(n=43)$.

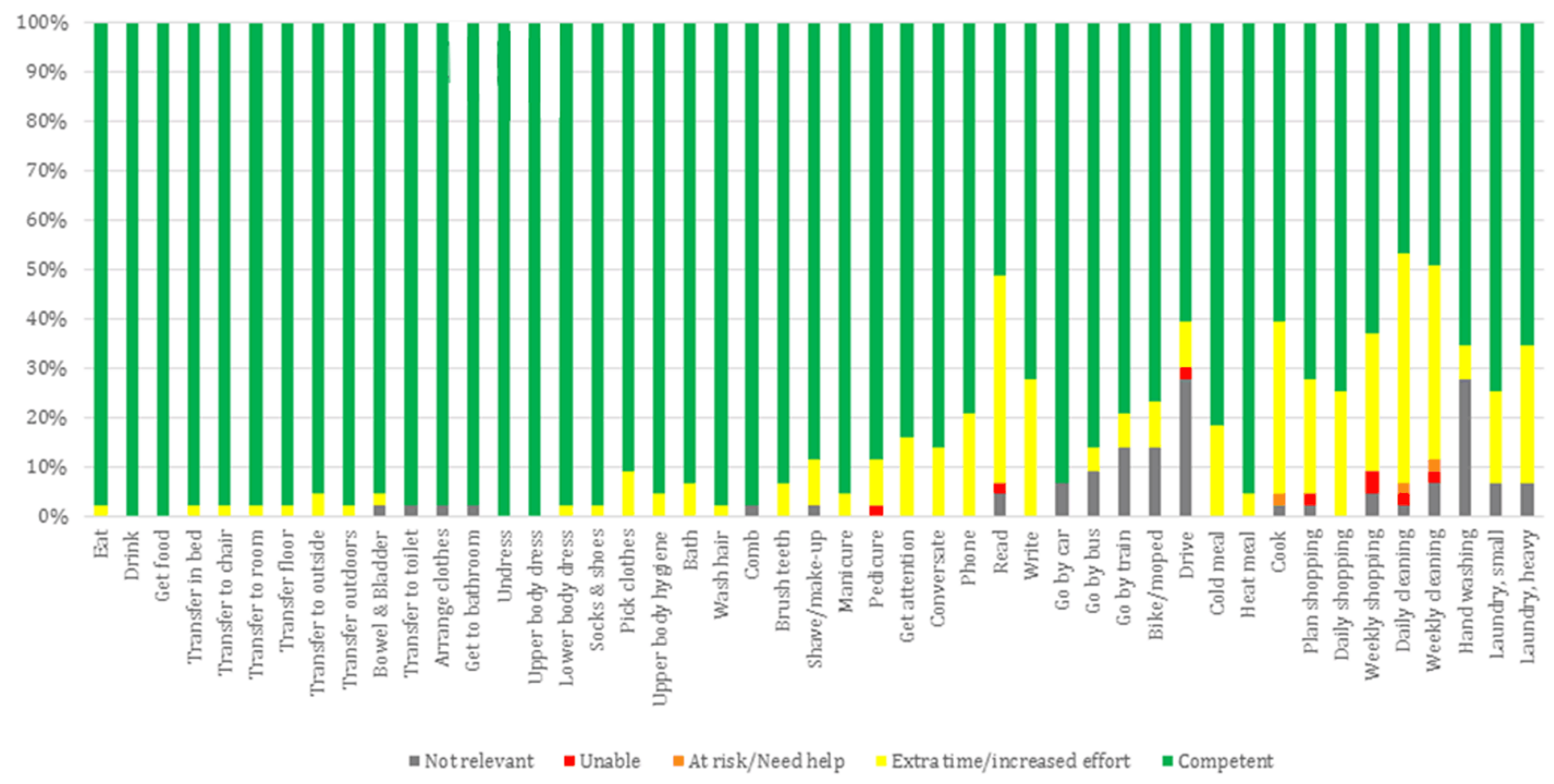

Figure 2: Self-reported Activities of Daily Living ability among patients with bipolar disorder. 


\section{EDORIUM Journals}
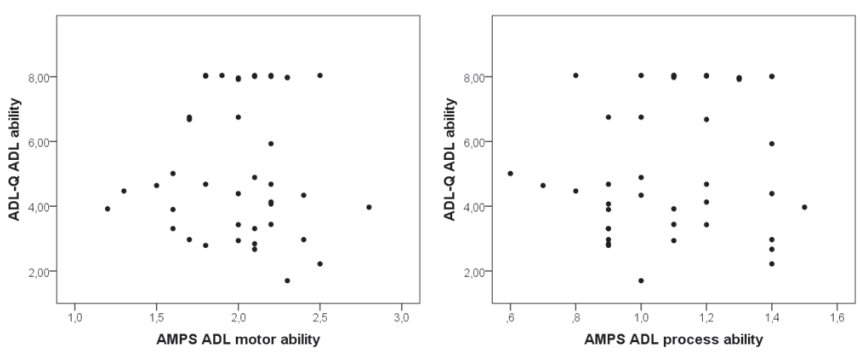

Figure 3: Correlations between measures of observed and selfreported Activities of Daily Living-questionnaire (ADL-Q) Activities of Daily Living ability among patients with bipolar disorder.

indicating increased physical effort, clumsiness or fatigue and potential safety risk during ADL task performance. Likewise, almost one third had an observed ADL process ability below the AMPS ADL process competence cut-off, showing decreased disorganization or undesirable use of time, space or objects and potential safety risk during ADL task performance. A minority (12\%) had observed ADL ability measures below both competence cut-offs. This supports previous studies which found decreased ADL ability in bipolar disorder patients despite heterogeneity both in terms of participants' clinical stage, age and instruments applied $[13-15,22]$. Finally, the observed decreased ADL process ability indicates that almost one third of the included participants may need assistance to live in the community, in line with previous studies using clinician ratings [13] or self-report [23]. Studies using AMPS on patients with schizophrenia have overall found a higher percentage from $17-72 \%$ that may need assistance to live in the community [37, 48, 49]. Similarly, studies on patients with major depression using AMPS have found that approximately $47 \%$ may need assistance to live in the community [37].

Most participants reported themselves as being competent within the PADL domain. However, in relation to reading (50\%) and writing by hand (30\%), participants did report the use of extra time or increased effort. This is not in line with a review by Ávila et al. [18], where only $13 \%$ of patients with bipolar disorder reported problems with reading. The participants in Ávila et al.'s study group had a higher mean age (47 years) than our study sample (median: 35 years, range: $19-58$ years) and a lower percentage of participants in education (6.7\% vs. $25.6 \%$ ). Thus, the proportion of patients with bipolar disorder in remission having writing and reading problems needs to be addressed within a larger group of participants.

Within the IADL domain, 20-50\% reported problems related to domestic tasks such as cooking, shopping, cleaning, washing clothes and driving. Two to four percent reported inability to perform some of the tasks or a safety risk. In comparison, the observed decreased ADL process ability indicates that almost one-third of the included participants may need assistance to live in the community. This difference may be due to the participants' focus on more obvious difficulties and not on the details of the quality of their performance: increased physical effort, clumsiness or fatigue and decreased disorganization or undesirable use of time, space, or objects. However, this is only a hypothesis and needs to be addressed in a future study. Our findings of self-reported decreased IADL ability among patients with bipolar disorder in remission are supported by other studies using clinical ratings [13] or observation-based assessments [14] of ADL.

Previous studies using observation-based in-home assessment have shown decreased ADL ability in elderly (age 50+) patients with bipolar disorder [16, 28, 50]. This is not surprising, as even healthy persons experience a progressive decrease in ADL ability from 50 years of age and older [12]. Therefore, one of the aims of this study was to compare the ADL ability of patients with bipolar disorder in remission with age-matched healthy persons. Overall, the participants had an observed ADL ability within age expectations but below what is expected for the average healthy age-matched person. We showed that the mean ADL motor ability in the present study sample was equivalent to that of healthy persons at 60-64 years and the mean $\mathrm{ADL}$ process ability was equivalent to healthy person older than 85 years [12]. Clinically, these observations point to a need for higher clinical awareness of daily hassles of patients with bipolar disorder and warrant more attention towards performing observations in their daily home surroundings.

We found no relation between self-reported and observation-based measures of ADL ability. This has not previously been addressed in patients with bipolar disorder in remission, but is in line with results from a study involving patients with depression [45]. However, previous studies involving assessment of cognition have shown a similar pattern of only weak correlations between subjective complaints and objective measures of cognitive impairment in patients with bipolar disorder [33, 5153]. This indicates an overall difference in the insider's (patient's) and outsider's (clinician's) perspectives within various domains and that self-reported and observationbased measures provide distinct but complementary information. Thus, when assessing ADL ability, the use of both types of assessments is recommended. The same applies for assessing cognition and ADL ability. We studied and reported on the correlation between ADL ability and cognitive impairment in a recent paper based on the same study sample. The results indicated that cognition and ADL ability are two distinct phenomena and therefore need to be assessed separately [34]. This is in line with the recommendations from a recent metaanalysis of the association between cognition and the ability to perform activities [17]. 


\section{EDORIUM Journals}

Edorium J Disabil Rehabil 2017;3:69-79.

Decker et al.

\section{Strengths and limitations}

The strength of our study was the use of observationbased assessment of ADL task performance based on a standardized instrument incorporating the international AMPS database with data from more than 150,000 individuals worldwide [11] which meets the concerns related to previously used assessments of ADL ability. Moreover, the use of both observation-based and selfreporting assessments that provided overall linear ADL measures is a strength. To the best of our knowledge, other assessments of ADL ability provide ordinal data, which has the drawback of not producing measures that can be used for parametric statistics.

As patients with bipolar disorder carry significant overall decreased functioning even during periods of remission [13], it is a strength in the study design that we only included patients in remission. Further, the patients were observed in their natural environment performing well-known tasks in the way they do in their everyday life. Other studies using observation-based assessments have used UCSD Performance-based Skills Assessment involving role-play $[14,15,25,28]$ or computerized shopping tasks [26]. The tasks in a roleplay assessment may not be familiar to the patient due, for example, to their cultural background. Both computerized tasks and role-play can be considered contrived assessments, as they do not take place in the patient's natural environment, which delimits the external validity of the studies [27, 28]. Based on observation of the participants in their home, we hypothesized that some may compensate for their cognitive impairment or illness with support from relatives and/or their ability to organize the environment. This fits in well with the ICF definition of functioning [6] and will need to be addressed in future studies. Furthermore, this hypothesis is supported by the results from another study indicating that contextual factors such as perceived social support appear to affect functioning within bipolar disorder in remission [9].

There are several limitations to this study: the main limitation was the rather small sample size, which means that the results can only be indicators of ADL ability within bipolar disorder in remission. Further, there is growing evidence of the influence of cognitive impairments on performance in everyday life within bipolar disorder [14, 15, 19, 28], which could implicate the need for objective cognitive impairment as an inclusion criterion instead of only subjective cognitive complaints. In particular as only weak correlations between subjective cognitive complaints and objective measures of cognitive impairment have been found [33, 51, 53, 54].

Finally, the lack of using assessments of well-being or quality of life to determine if there is an association between measures of ADL ability and subjective measures of well-being is a limitation.

\section{CONCLUSION}

Patients with bipolar disorder in remission reporting subjective cognitive complaints have both observed Activities of Daily Living (ADL) motor and ADL process ability below the mean of healthy persons of the same age. There can be concerns for safe task performance and almost one-third may need assistance to live in the community. Although both observation-based and the self-reporting assessment did show decreased ADL ability there was a trend towards patients evaluating themselves as more competent than what was observed. As no association between self-reported and observation-based ADL ability was found, both clinical practice and future studies are recommended to address both areas and use validated observation-based in-home assessment tools to evaluate the outcomes of intervention programs aiming to improve functioning for the most impaired third of patients with bipolar disorder.

$* * * * * * * * *$

\section{Author Contributions}

Lone Decker - Substantial contributions to conception and design, Acquisition of data, Analysis and interpretation of data, Drafting the article, Revising it critically for important intellectual content, Final approval of the version to be published

Conny Träger - Acquisition of data, Analysis and interpretation of data, Drafting the article, Revising it critically for important intellectual content, Final approval of the version to be published

Kamilla Miskowiak - Substantial contributions to conception and design, Drafting the article, Revising it critically for important intellectual content, Final approval of the version to be published

Eva Ejlersen Wæhrens - Substantial contributions to conception and design, Acquisition of data, Analysis and interpretation of data, Drafting the article, Revising it critically for important intellectual content, Final approval of the version to be published

Maj Vinberg - Substantial contributions to conception and design, Acquisition of data, Analysis and interpretation of data, Drafting the article, Revising it critically for important intellectual content, Final approval of the version to be published

\section{Guarantor}

The corresponding author is the guarantor of submission.

\section{Conflict of Interest}

This study was supported by an independent grant from the Lundbeck Foundation, Denmark. The funding organization had no role in the design and conduct of the study; collection, management, analysis, and interpretation of the data; and preparation, review, or 


\section{EDORiUM Journals}

approval of the manuscript; and decision to submit the manuscript for publication.

Lone Decker has within the past three years received speaker's honoraria from Lundbeck. Conny Träger and Eva Ejlersen Wæhrens have no conflicts of interest. Maj Vinberg has within the past three years been a consultant for Lundbeck and AstraZeneca. Kamilla W. Miskowiak reports having received consultancy fees from Lundbeck and Allergan in the past three years.

\section{Copyright}

(C) 2017 Lone Decker et al. This article is distributed under the terms of Creative Commons Attribution License which permits unrestricted use, distribution and reproduction in any medium provided the original author(s) and original publisher are properly credited. Please see the copyright policy on the journal website for more information.

\section{REFERENCES}

1. Global burden of disease study 2013 Collaborators. Global, regional, and national incidence, prevalence, and years lived with disability for 301 acute and chronic diseases and injuries in 188 countries, 1990-2013: A systematic analysis for the global burden of disease study 2013. Lancet 2015 Aug 22;386(9995):743-800.

2. Whiteford HA, Ferrari AJ, Degenhardt L, Feigin V, Vos T. The global burden of mental, neurological and substance use disorders: An analysis from the global burden of Disease Study 2010. PLoS One 2015 Feb 6;10(2):e0116820.

3. Akiskal HS, Bourgeois ML, Angst J, Post R, Möller $\mathrm{H}$, Hirschfeld R. Re-evaluating the prevalence of and diagnostic composition within the broad clinical spectrum of bipolar disorders. J Affect Disord 2000 Sep;59 Suppl 1:S5-S30.

4. Merikangas KR, Jin R, He JP, et al. Prevalence and correlates of bipolar spectrum disorder in the world mental health survey initiative. Arch Gen Psychiatry 2011 Mar;68(3):241-51.

5. Avila CC, Cabello M, Cieza A, Vieta E, Ayuso-Mateos JL. Functioning and disability in bipolar disorders: A systematic review of literature using the ICF as a reference. Bipolar Disord 2010 Aug;12(5):473-82.

6. World Health Organization. International classification of functioning, disability and health. Geneva: WHO; 2001.

7. Ustün B, Kennedy C. What is "functional impairment"? Disentangling disability from clinical significance. World Psychiatry 2009 Jun;8(2):82-5.

8. Ayuso-Mateos JL, Avila CC, Anaya C, Cieza A, Vieta E; Bipolar disorders Core Sets expert group. Development of the international classification of functioning, disability and health core sets for bipolar disorders: Results of an international consensus process. Disabil Rehabil 2013;35(25):2138-46.

9. Sanchez-Moreno J, Martinez-Aran A, Gadelrab HF, et al. The role and impact of contextual factors on functioning in patients with bipolar disorder. Disabil Rehabil 2010;32 Suppl 1:S94-S104.

10. Goldberg JF, Chengappa KN. Identifying and treating cognitive impairment in bipolar disorder. Bipolar Disord 2009 Jun;11 Suppl 2:123-37.

11. Martínez-Arán A, Vieta E, Reinares M, et al. Cognitive function across manic or hypomanic, depressed, and euthymic states in bipolar disorder. Am J Psychiatry 2004 Feb;161(2):262-70.

12. Fisher A, Jones K. Assessment of motor and process skills. Vol. 1: Development, standardization, and administration manual. Fort Collins: Three Star Press; 2012.

13. Rosa AR, Franco C, Martínez-Aran A, et al. Functional impairment in patients with remitted bipolar disorder. Psychother Psychosom 2008;77(6):390-2.

14. Henry BL, Minassian A, Perry W. Everyday functional ability across different phases of bipolar disorder. Psychiatry Res 2013 Dec 30;210(3):850-6.

15. Bowie CR, Depp C, McGrath JA, et al. Prediction of real-world functional disability in chronic mental disorders: A comparison of schizophrenia and bipolar disorder. Am J Psychiatry 2010 Sep;167(9):1116-24.

16. Gildengers AG, Butters MA, Chisholm D, et al. Cognitive functioning and instrumental activities of daily living in late-life bipolar disorder. Am J Geriatr Psychiatry 2007 Feb;15(2):174-9.

17. Depp CA, Mausbach BT, Harmell AL, et al. Metaanalysis of the association between cognitive abilities and everyday functioning in bipolar disorder. Bipolar Disord 2012 May;14(3):217-26.

18. Ávila CC, Cieza A, Anaya C, Ayuso-Mateos JL. The patients' perspective on relevant areas and problems in the bipolar spectrum disorder: Individual interviews using the international classification of functioning, disability and health as a reference tool. Am J Phys Med Rehabil 2012 Feb;91(13 Suppl 1):S181-8.

19. Martinez-Aran A, Vieta E, Torrent C, et al. Functional outcome in bipolar disorder: The role of clinical and cognitive factors. Bipolar Disord 2007 Feb-Mar;9(12):103-13.

20. Sanchez-Moreno J, Martinez-Aran A, et al. TabarésSeisdedos R, Torrent C, Vieta E, Ayuso-Mateos JL. Functioning and disability in bipolar disorder: An extensive review. Psychother Psychosom 2009;78(5):285-97.

21. Pope M, Dudley R, Scott J. Determinants of social functioning in bipolar disorder. Bipolar Disord 2007 Feb-Mar;9(1-2):38-44.

22. Simon GE, Bauer MS, Ludman EJ, Operskalski BH, Unützer J. Mood symptoms, functional impairment, and disability in people with bipolar disorder: Specific effects of mania and depression. J Clin Psychiatry 2007 Aug;68(8):1237-45.

23. Levine J, Chengappa KN, Brar JS, Gershon S, Kupfer DJ. Illness characteristics and their association with prescription patterns for bipolar I disorder. Bipolar Disord 2001 Feb;3(1):41-9.

24. Patterson TL, Mausbach BT. Measurement of functional capacity: A new approach to understanding functional differences and real-world behavioral adaptation in those with mental illness. Annu Rev Clin Psychol 2010;6:139-54. 


\section{EDORiUM Journals}

25. Mausbach BT, Harvey PD, Pulver AE, et al. Relationship of the brief UCSD performance-based skills assessment (UPSA-B) to multiple indicators of functioning in people with schizophrenia and bipolar disorder. Bipolar Disord 2010 Feb;12(1):45-55.

26. Laloyaux J, Pellegrini N, Mourad H, et al. Performance on a computerized shopping task significantly predicts real world functioning in persons diagnosed with bipolar disorder. Psychiatry Res 2013 Dec 15;210(2):465-71.

27. Moore DJ, Palmer BW, Patterson TL, Jeste DV. A review of performance-based measures of functional living skills. J Psychiatr Res 2007 Jan-Feb;41(12):97-118.

28. Depp CA, Mausbach BT, Eyler LT, et al. Performancebased and subjective measures of functioning in middle-aged and older adults with bipolar disorder. J Nerv Ment Dis 2009 Jul;197(7):471-5.

29. World Health Organization. International Statistical Classification of Diseases and Related Health Problems: 10th Revision (ICD-10). Geneva: WHO; 1996.

30. Hamilton M. Development of a rating scale for primary depressive illness. Br J Soc Clin Psychol 1967 Dec;6(4):278-96.

31. Young RC, Biggs JT, Ziegler VE, Meyer DA. A rating scale for mania: Reliability, validity and sensitivity. $\mathrm{Br}$ J Psychiatry 1978 Nov;133:429-35.

32. Rosa AR, Mercadé C, Sánchez-Moreno J, et al. Validity and reliability of a rating scale on subjective cognitive deficits in bipolar disorder (COBRA). J Affect Disord 2013 Aug 15;150(1):29-36.

33. Jensen JH, Støttrup MM, Nayberg E, et al. Optimising screening for cognitive dysfunction in bipolar disorder: Validation and evaluation of objective and subjective tools. J Affect Disord 2015 Nov 15;187:109.

34. Trager C, Decker L, Waehrens EE, Knorr U, Miskowiak $\mathrm{K}$, Vinberg M. Influences of patient informed cognitive complaints on activities of daily living in patients with bipolar disorder. An exploratory cross-sectional study. Psychiatry Res 2017 Mar;249:268-74.

35. Fisher A, Jones K. Assessment of motor and process skills. Vol. 2. User manual. Fort Collins: Three Star Press; 2014.

36. OT Assessment Package Version 3.00. Fort Collins: Three Star Press, inc. 2014

37. Girard C, Fisher AG, Short MA, Duran L. Occupational performance differences between psychiatric groups. Scand J Occup Ther 1999;6(3):119-26.

38. McNulty MC, Fisher AG. Validity of using the assessment of motor and process skills to estimate overall home safety in persons with psychiatric conditions. Am J Occup Ther 2001 NovDec;55(6):649-55.

39. Moore K, Merritt B, Doble SE. ADL skill profiles across three psychiatric diagnoses. Scand J Occup Ther 2010;17(1):77-85.

40. Oakley F, Khin NA, Parks R, Bauer L, Sunderland T. Improvement in activities of daily living in elderly following treatment for post-bereavement depression. Acta Psychiatr Scand 2002 Mar;105(3):231-4.
41. Pan AW, Fisher AG. The assessment of motor and process skills of persons with psychiatric disorders. Am J Occup Ther 1994;48(9):775-80.

42. Waehrens EE. Measuring quality of occupational performance based on self-report and observation: Development and validation of instruments to evaluate ADL task performance. Department of community medicine and rehabilitation, Occupational Therapy, Umeå University. 2010.

43. Wæhrens EE, Bliddal H, Danneskiold-Samsøe B, Lund H, Fisher AG. Differences between questionnaire- and interview-based measures of activities of daily living (ADL) ability and their association with observed ADL ability in women with rheumatoid arthritis, knee osteoarthritis, and fibromyalgia. Scand J Rheumatol 2012 Mar;41(2):95-102.

44. Bendixen HJ, Wæhrens EE, Wilcke JT, Sørensen LV. Self-reported quality of ADL task performance among patients with COPD exacerbations. Scand J Occup Ther 2014 Jul;21(4):313-20.

45. Nielsen KT, Waehrens EE. Occupational therapy evaluation: Use of self-report and/or observation? Scand J Occup Ther 2015;22(1):13-23.

46. IBM CU. Statistical package for the Social Sciences Version 22.0. [Available at: https://www-01.ibm. com/support/docview.wss?uid=swg21646821]

47. Linacre J. WINSTEPS $®$ Rash measurement computer program. Version 3.68.2. Chicago IL, 2009.

48. Ayres H, John AP. The assessment of motor and process skills as a measure of ADL ability in schizophrenia. Scand J Occup Ther 2015;22(6):4707 .

49. Fossey E, Harvey C, Plant G, Pantelis C. Occupational performance of people diagnosed with schizophrenia in supported housing and outreach programmes in australia. British Journal of Occupational Therapy 2016;69(9):409-19.

50. Gildengers AG, Chisholm D, Butters MA, Twoyear course of cognitive function and instrumental activities of daily living in older adults with bipolar disorder: Evidence for neuroprogression? Psychol Med 2013 Apr;43(4):801-11.

51. Burdick KE, Ketter TA, Goldberg JF, Calabrese JR. Assessing cognitive function in bipolar disorder: Challenges and recommendations for clinical trial design. J Clin Psychiatry 2015 Mar;76(3):e342-50.

52. Demant KM, Vinberg M, Kessing LV, Miskowiak KW. Effects of short-term cognitive remediation on Cognitive dysfunction in partially or fully remitted individuals with bipolar disorder: Results of a randomised controlled trial. PLoS One 2015 Jun 12;10(6):e0127955.

53. van der Werf-Eldering $\mathrm{MJ}$, Burger $\mathrm{H}$, Jabben $\mathrm{N}$, Holthausen EA, Aleman A, Nolen WA. Is the lack of association between cognitive complaints and objective cognitive functioning in patients with bipolar disorder moderated by depressive symptoms? J Affect Disord 2011 Apr;130(1-2):306-11.

54. Svendsen AM, Kessing LV, Munkholm K, Vinberg $\mathrm{M}$, Miskowiak KW. Is there an association between subjective and objective measures of cognitive function in patients with affective disorders? Nord J Psychiatry 2012 Sep;66(4):248-53. 


\section{EDORiUM Journals}

Edorium J Disabil Rehabil 2017;3:69-79.

www.edoriumjournals.com/ej/dr

Access full text article on other devices

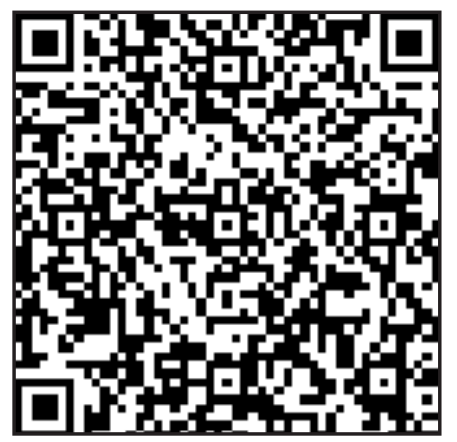

Access PDF of article on other devices

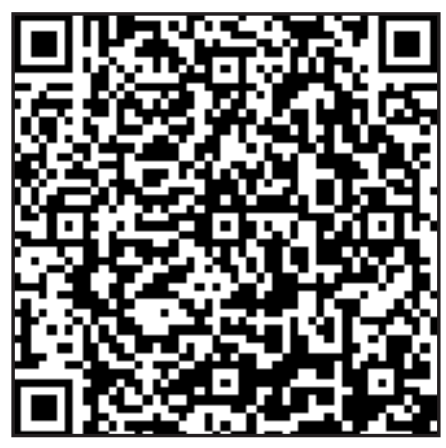

